\title{
Utility of TMPRSS4 as a Prognostic Biomarker and Potential Therapeutic Target in Patients with Gastric Cancer
}

\author{
Hirofumi Tazawa ${ }^{1}$ (D) $\cdot$ Takahisa Suzuki $^{1,2} \cdot$ Akihisa Saito $^{3} \cdot$ Akira Ishikawa $^{3} \cdot$ Toshiaki Komo $^{1} \cdot$ Haruki Sada $^{1}$. \\ Norimitsu Shimada ${ }^{1} \cdot$ Naoto Hadano $^{1} \cdot$ Takashi Onoe $^{1} \cdot$ Takeshi Sudo $^{1} \cdot$ Yosuke Shimizu $^{1} \cdot$ Kazuya Kuraoka $^{3}$. \\ Hirotaka Tashiro ${ }^{1,2}$
}

Received: 28 May 2021 / Accepted: 14 July 2021 / Published online: 11 August 2021

(C) 2021 The Author(s)

\begin{abstract}
Background Transmembrane serine protease 4 (TMPRSS4) belongs to the family of type II transmembrane serine proteases that are known to be upregulated in many malignant tumors. However, there is a paucity of studies documenting the clinical impact and biological effects of TMPRSS4 on gastric cancer (GC) patients who underwent surgery.

Methods Tissues samples were obtained from 105 patients with GC who underwent gastrectomy followed by adjuvant chemotherapy, excluding those at stage I. The expression of TMPRSS4 was examined through immunohistochemical analysis. The association between TMPRSS4 expression and clinico-pathological features as well as prognosis was assessed. Moreover, the effects of TMPRSS4 expression on cell migration and sensitivity to 5-FU were investigated.

Results The expression rate of TMPRSS4 was $56.3 \%$ (59/105) in GC cases. The expression of TMPRSS4 was positively correlated with the depth of tumor $(\mathrm{T})$ and venous $(\mathrm{V})$ invasion. The 5 -year overall survival (OS) and recurrence-free survival (RFS) rates of the TMPRSS4-positive group was significantly lower than that of the TMPRSS4-negative group ( $p=0.0001$ and $p=0.005$, respectively). Especially, there was significant differences in OS and RFS of patients with stage III cancer between the two groups ( $p=0.0064$ and 0.012 , respectively). Multivariate analysis demonstrated that TMPRSS4 expression and the stage of cancer were crucial prognostic factors for RFS. TMPRSS4-silenced GC cells exhibited increased sensitivity to 5-FU when compared with the non-specific control siRNA-transfected cells.

Conclusion TMPRSS4 can be considered as a potential prognostic biomarker, especially for stage III, and a promising therapeutic target for GC.
\end{abstract}

Keywords Gastric cancer · TMPRSS4 · Biomarker

Hirofumi Tazawa

tazawa.hirofumi.tc@mail.hosp.go.jp

Hirotaka Tashiro

tashiro.hirotaka.zn@mail.hosp.go.jp

1 Department of Surgery, Kure Medical Center - Chugoku Cancer Center, 737-0023, 3-1, Kure, Hiroshima, Japan

2 Department of Gastroenterological and Transplant Surgery, Graduate School of Biochemical and Health Sciences, Hiroshima University, Hiroshima, Japan

3 Department of Diagnostic Pathology, Kure Medical Center · Chugoku Cancer Center, Kure, Hiroshima, Japan

\section{Introduction}

Surgery is considered as the main treatment method for operable gastric cancer (GC). However, appreciable patients at stages II and III undergo recurrence. Therefore, various adjuvant chemotherapy methods have been developed and tested to prevent postoperative recurrence. ${ }^{1}$ In 2007, the Adjuvant Chemotherapy Trial of S-1 for GC (ACTS-GC) demonstrated that surgery in addition to a 12 months of postoperative adjuvant chemotherapy with S-1 improved the overall survival (OS) and recurrence-free survival (RFS) rates compared to surgery alone for patients at stages II and III of GC. ${ }^{2}, 3$ However, the 5-year OS rate in patients at stages IIIA and IIIB were 67.1 and $50.2 \%$ in the S-1 group, respectively, indicating the need for further improvement. Therefore, in 2019, through additional therapy of docetaxel to the S-1 group, 
Japan Clinical Cancer Research Organization (JACRO) GC07 improved the prognosis of gastric patients at stage IIII: the 3 -year RFS rate in patients at stage III was $66 \%$ in the S-1 plus docetaxel group and $50 \%$ in the S-1 alone group. However, the data related to OS was not presented.

Serine proteases are involved in the degradation of basement membrane and extracellular matrix, which allows tumor cells to invade the surrounding tissue. Proteases are involved in the proliferation, migration, invasion, and angiogenesis of cancer cells. Type II transmembrane serine proteases (TTSPs) are a novel subfamily of serine protease and are known to be upregulated in malignant tumors. ${ }^{5} 6$ Transmembrane serine protease 4 (TMPRSS4) belongs to a family of TTSPs. It is overexpressed in many cancer types, including pancreatic cancer, colon cancer, lung cancer, ovary cancer, hepatocellular carcinoma, and $\mathrm{GC}^{7}{ }^{7}-12$ The expression of TMPRSS4 4 is associated with the epithelial to mesenchymal transition (EMT) and upregulates integrin- $\alpha 5$ to induce invasiveness. ${ }^{13}, 14$ Additionally, TMPRSS4 regulates urokinase-type plasminogen activator (uPA) via a dual mechanism through increasing the gene expression and processing of pro-uPA into its active form, which leads to an enhanced invasion. ${ }^{15}$

Recently, the overexpression of TMPRSS4 has been shown to be associated with poor prognosis in GC patients at stages II and III post-surgery. ${ }^{16}$ However, a few studies have documented the impact of TMPRSS4 on adjuvant chemotherapy in GC patients who underwent surgery. The effect of TMPRSS4 on the sensitivity of GC cells to chemotherapy was not examined yet.

In the present study, we examined the expression of TMPRSS4 in GC tissues and its correlation with clinicopathological parameters and prognosis in GC patients undergoing adjuvant chemotherapy following surgery. We also investigated the effect of TMPRSS4 expression on the sensitivity to chemotherapy in GC cells.

\section{Materials and Methods}

\section{Patients}

Between January 2012 and January 2015, 105 patients underwent resection for GC at the Department of Surgery, Kure Medical Center and Chugoku Cancer Center, National Hospital Organization. All patients received R0 resection with more than D1 plus or D2 lymph node dissection according to the previously outlined guideline. ${ }^{17}$ Patients with histologically confirmed stage I GC did not receive adjuvant chemotherapy. However, patients with histologically confirmed stage II or III GC received adjuvant chemotherapy consisting of S-1 ( 80 to $120 \mathrm{mg}$ per day) for 4 weeks, followed by 2 weeks of rest. This 6 -week cycle was repeated for 1 year, according to the previously outlined guidelines. ${ }^{2}$ Patients with histologically confirmed stage IV GC received adjuvant chemotherapy consisting of S-1 in addition to cisplatin. No patients received chemotherapy before the operation.

The patient's medical records were reviewed retrospectively, and data were collected. Following this step, OS and RFS were analyzed. Tumors were classified based on the Japanese Classification of Gastric Carcinoma, $14^{\text {th }}$ edition. ${ }^{18}$ This retrospective study (approval number: 2019-93) was reviewed and approved by the Institution Review Board of Kure Medical Center.

\section{Immunohistochemical Analysis}

Immunohistochemical analysis was carried out following the manufacturer's instructions (DAKO). Four-micrometer-thick tissue sections were incubated with the primary rabbit antiTMPRSS4 antibody (1:200; abl50595, Abcam, Cambridge, UK) overnight at $4{ }^{\circ} \mathrm{C}$ followed by the incubation with the secondary antibody. All immunohistochemistry results were determined by two independent pathologists (A.S., K.K.) blinded to this study. The percentage of positively stained tumor cells was scored as follows: TMPRSS4-postive, 50\% positive and more than $50 \%$ positive cells; TMPRSS4-negative, less than $50 \%$ positive cells.

\section{Cell Culture and Reagents}

The two human GC cell lines used in this study are as follows: NUGC3 (from JCRB Cell Bank), and MKN45 (from RIKEN Cell Bank). All cell lines were cultured in RPMI-1640 medium (Nacalai Tesque, Kyoto, Japan) supplemented with $10 \%$ fetal bovine serum (Life Technologies, Carlsbad, CA) at 37 ${ }^{\circ} \mathrm{C}$ in a humidified atmosphere supplied with $5 \% \mathrm{CO}_{2}$.

\section{RNA Interference}

\section{siRNA Transfection}

Cells were transfected with TMPRSS4-siRNA MISSIONsiRNA (\#SASI_Hs02_00317341, \#SASI_Hs02_00317342, and \#SASI_Hs02_00317343; Sigma-Aldrich, St. Louis, MO) using the Lipofectamine RNAiMAX reagent (Invitrogen; Thermo Fisher Scientific, Waltham, MA), according to the manufacturer's instructions. The control siRNA MISSON-siRNA Universal Negative Control (Sigma-Aldrich) was used as a negative control.

\section{Quantitative Real-Time PCR (qPCR)}

Total RNA was extracted using NucleoSpin RNA (Takara Bio, Shiga, Japan) and then was reverse transcribed into cDNA using PrimerScript RT MasterMix (Takara Bio) according to the manufacturer's protocol. The resulting cDNA 
was amplified using TB Green Premix Ex Taq II (Takara Bio). The expression of the target gene was quantified using the comparative cycle threshold method. The primer sequences used were as follows: TMPRSS4 forward, 5'-ACTA CTGAGCCTGGCGAGTA-3', and reverse, 5'-TTGA CACAGTGCTCCTCGTC-3', and GAPDH forward, 5'CAACGGATTTGGTCGTATTGG-3', and reverse, 5'CCATGGGTGGAATCATATTGG-3'.

\section{Western Blotting}

Cells were washed with PBS and lysed with Cell Lysis Buffer (Cell Signaling, Beverly, MA) containing PMSF (Cell Signaling). Equal amounts of sample proteins were denatured through boiling for $10 \mathrm{~min}$ in SDS-sample buffer containing $1 \%$ beta-mercaptoethanol. The proteins were then electrophoresed on an SDS-polyacrylamide gel and transferred onto a PVDF membrane (Bio-Rad, Hercules, CA). The membrane was blocked using 5\% skim milk prepared in Tris-buffered saline containing $0.05 \%$ Tween 20 for $1 \mathrm{~h}$, and thereafter, it was incubated with a primary antibody overnight. The primary antibodies used are as follows: anti-TMPRSS4 rabbit polyclonal antibody (1:2000;11283-1, Proteintech) and anti-beta actin mouse monoclonal antibody (1:10,000; A5441, SigmaAldrich). Proteins were then visualized with an anti-mouse and rabbit IgG horseradish peroxidase-conjugated secondary antibody (1:3,000; Cell Signaling) using the ECL Western Blotting Detection System (Bio-Rad Technologies).

\section{Wound-Healing Assay}

Cells were transfected with the above-indicated siRNAs for 72 h. Cell monolayers were scratched and then incubated in medium with $10 \%$ FBS. About 24/48 h after scratching, the percent reduction of the scratched area was evaluated using BZX700 (Keyence, Osaka, Japan).

\section{Proliferation Assay}

Cells were seeded at a concentration of 4,500 cells (NUGC3), and 3,000 cells (MKN45) per well in a 96-well plate and transfected with the above-indicated siRNAs for $72 \mathrm{~h}$. After transfection, the cells were exposed to different concentrations of 5-fluorouracil (5-FU) for $72 \mathrm{~h}$. Thereafter, cell proliferation was evaluated using the Cell Counting Kit-8 (CCK-8, Dojindo, Kumamoto, Japan). The optical absorbance of samples was measured using the fluorescence microplate reader MULTISKAN GO (Thermo Fisher Scientific, Fremont, CA) at a wavelength of $450 \mathrm{~nm}$ and applied to the following formula: Relative cell viability $=$ Number of cells treated with drugs/Number of cells treated with vehicle. 5-FU was purchased from Towa pharmaceutical (Osaka, Japan).

\section{Statistical Analysis}

The continuous variables were expressed as median (range) and were analyzed using Mann-Whitney $U$-test. The categorical variables and postoperative courses were compared using $\chi^{2}$ tests with Yates correction. OS and RFS after surgical resection were compared in each group using Kaplan-Meier method and log-rank test. Univariate and multivariate analyses were performed using Cox regression test. The difference was considered statistically significant if the $p$ value was less than 0.05. Statistical analyses were performed using the SPSS statistical software version 16 (Chicago, Illinois, USA).

\section{Results}

\section{Overexpression of TMPRSS4 in GC Tissues}

TMPRSS4-positive staining was mainly observed in the cytoplasm of GC cell using immunohistochemical analysis (Fig. 1). The expression rate of TMPRSS4 was $56.3 \%(59 / 105)$ in all GC cases (Table 1).

\section{Association Between Clinico-pathological Features and TMPRSS4 Positivity}

We evaluated the association of TMPRSS4 positivity with clinico-pathological features (Table 1). Regarding the tumor location, the TMPRSS4-positive cells were higher in the proximal site than the distal site of stomach without any significance. The expression of TMPRSS4 was positively and significantly correlated with the depth of tumor invasion (T) and venous invasion (v), but not with the age, gender, tumor size, lymph node metastasis $(\mathrm{N})$, histological type, and stages of the tumor.

\section{Association Between the Expression of TMPRSS4 and the Outcomes of Patients After Gastrectomy}

The 5-year OS rate of the TMPRSS4-positive group was significantly lower than that of the TNMPRSS4-negative group ( $51 \%$ vs $87 \%, p=0.0001$ ). Moreover, the 5 -year RFS rate was also significantly lower in the TMPRSS4-positive group compared with the TMPRSS4-negative group (43\% vs $83 \%$, $p=0.005$ ) (Fig. 2a, b). Next, the univariate and multivariate analyses were conducted using Cox proportional hazard model to evaluate the influence of TMPRSS4 expression and pathological factors on the prognosis of GC patients who underwent gastrectomy. In regard to OS, the univariate analysis revealed that TMPRSS4, tumor size, deeper tumor invasion, lymph node metastasis, venous invasion, lymphatic invasion, invasion type, and stages of tumor were all significant prognostic factors for OS. However, the multivariate analysis 
Table 1 Correlation between TMPRSS4 and clinical features

\begin{tabular}{llll}
\hline Variables & \multicolumn{2}{l}{ TMPRSS4 expression } & \\
\cline { 2 - 4 } & $\begin{array}{l}\text { Negativity } \\
N=46\end{array}$ & Positivity \\
& & $N=59$ & \\
\hline Age & $66.7(38-87)$ & $71.5(45-91)$ & 0.2396 \\
Gender (male/female) & $32 / 14$ & $40 / 19$ & 0.9302 \\
Tumor location (J/U/M/L) & $2 / 4 / 20 / 18$ & $5 / 11 / 20 / 23$ & 0.0996 \\
Tumor size & $53.3(7-200)$ & $62.1(15-167)$ & 0.6574 \\
pStaging (I/II/II/IV) & $20 / 10 / 14 / 2$ & $11 / 16 / 24 / 8$ & 0.1876 \\
Depth of tumor invasion (T) (1/2/3/4) & $18 / 6 / 16 / 6$ & $8 / 5 / 34 / 12$ & 0.0258 \\
Lymph node metastasis (N) (0/1/2/3) & $19 / 7 / 8 / 12$ & $17 / 12 / 10 / 20$ & 0.6475 \\
Lymphatic invasion (ly) (0/1/2/3) & $24 / 10 / 6 / 6$ & $18 / 14 / 11 / 15$ & 0.2152 \\
Venous invasion (v) (0/1/2/3) & $34 / 9 / 3 / 0$ & $26 / 22 / 8 / 3$ & 0.0108 \\
Histology (tub/por/other) & $20 / 22 / 4$ & $18 / 35 / 6$ & 0.6868 \\
Tumor infiltrative pattern (IFN) (a/b/c) & $14 / 14 / 9$ & $13 / 19 / 19$ & 0.1094 \\
Lauren classification (i/m/d) & $13 / 17 / 8$ & $18 / 21 / 12$ & 0.2033 \\
\hline
\end{tabular}

$J$ esophagogastric junction, $U$ upper third, $M$ middle third, $L$ lower third, $i$ intestinal type, $m$ mixed type, $d$ diffuse type revealed that the stages of tumor and venous invasion were significant prognostic factors for OS, but TMPRSS4 was not a significant prognostic factor for OS (Table 2). In regard to RFS, the univariate analysis revealed that the gender, Lauren's pathological type, TMPRSS4, tumor size, deeper tumor invasion, lymph node metastasis, venous invasion, lymphatic invasion, invasion type, and stages of tumor were all significant prognostic factors for RFS. Moreover, the multivariate analysis revealed that TMPRSS4 and the stages of tumor were also significant prognostic factors for RFS (Table 3).
In terms of clinical stages, there was significant differences in OS and RFS of patients with stage III GC between the two groups $(p=0.0064$ and $p=0.012$, respectively): the 5-year OS rates of TMPRSS4-positive and TMPRSS4-negative groups were $38 \%$ and $85 \%$, respectively, and the 5-year RFS rates of TMPRSS4-positive and TMPRSS4-negative group were $18 \%$ and $67 \%$, respectively (Fig. 3a, b). For the patients at stage I, the 5year OS rates in both groups were $100 \%$ and $100 \%$, respectively, and there was no significant difference in the RFS rates between the two groups, although TMPRSS4-
Fig. 1 Immunohistochemical staining of TMPRSS4 in GC lesions and non-cancerous tissues. a TMPRSS4-negative non-cancerous gastric tissues. $\mathbf{b}$ TMPRSS4-negative GC tissues with moderately differentiated adenocarcinoma. c TMPRSS4positive GC tissues with moderately differentiated adenocarcinoma. d TMPRSS4positive GC tissues with poorly differentiated adenocarcinoma (original magnification, $40 \times$ ). Scale bar indicates $100 \mu \mathrm{m}$. a

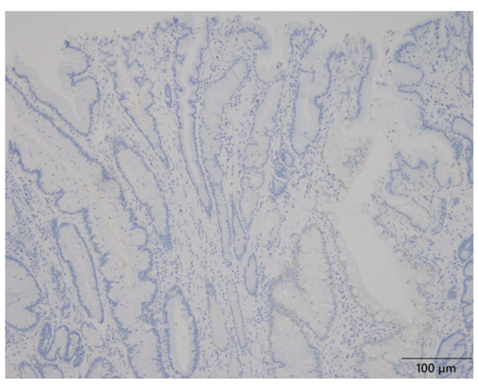

C

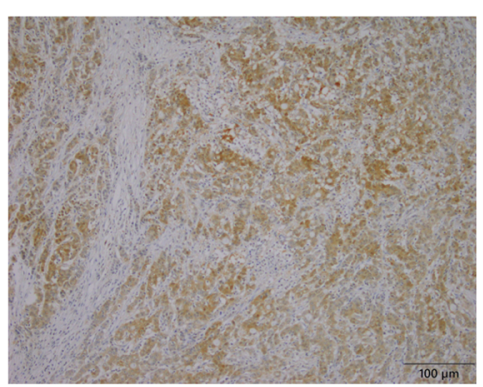

b

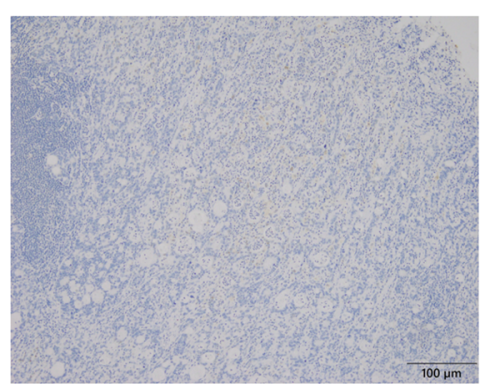

d

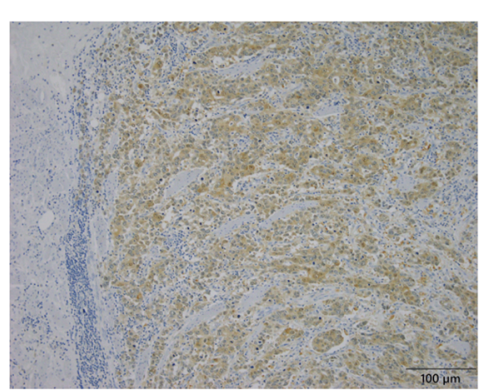


Table 2 Univariate analyses of OS and RFS in patients with gastric cancer

\begin{tabular}{|c|c|c|c|c|c|}
\hline \multirow[t]{2}{*}{ Variables } & \multirow[t]{2}{*}{ Number of patients } & \multicolumn{2}{|l|}{ OS } & \multicolumn{2}{|l|}{ RFS } \\
\hline & & 5 -year survival rate $(\%)$ & $p$ value & 5 -year survival rate $(\%)$ & $p$ value \\
\hline Age & & & 0.713 & & 0.2158 \\
\hline $70<$ & & 68.9 & & 65.8 & \\
\hline $70 \geq$ & & 65.8 & & 57.0 & \\
\hline Gender & & & 0.4 & & 0.0229 \\
\hline Male & 72 & 64.6 & & 53.0 & \\
\hline Female & 33 & 73.4 & & 82.1 & \\
\hline TMPRSS4 & & & 0.0001 & & 0.0005 \\
\hline Positivity & 59 & 52.0 & & 44.9 & \\
\hline Negativity & 46 & 88.0 & & 81.2 & \\
\hline Tumor location & & & 0.106 & & 0.1842 \\
\hline Junction & 7 & 71.4 & & 71.4 & \\
\hline Upper & 17 & 43.8 & & 40.0 & \\
\hline Middle & 40 & 77.3 & & 71.0 & \\
\hline Lower & 41 & 68.1 & & 59.1 & \\
\hline Tumor size & & & 0.0019 & & 0.0334 \\
\hline $52<$ & & 53.4 & & 73.5 & \\
\hline $52 \geq$ & & 83.9 & & 47.8 & \\
\hline pStage & & & 0.0001 & & 0.0001 \\
\hline I & 31 & 100.0 & & 90.3 & \\
\hline II & 25 & 79.0 & & 63.3 & \\
\hline II & 39 & 49.1 & & 36.4 & \\
\hline IV & 10 & 10.0 & & & \\
\hline Depth of tumor invasion(T) & & & 0.0001 & & 0.0003 \\
\hline $\mathrm{T} 1$ & 28 & 96.3 & & 89.3 & \\
\hline $\mathrm{T} 2$ & 11 & 77.8 & & 70.0 & \\
\hline $\mathrm{T} 3$ & 49 & 69.1 & & 51.1 & \\
\hline $\mathrm{T} 4$ & 17 & 11.7 & & 20.0 & \\
\hline Lymph node metastasis $(\mathrm{N})$ & & & 0.0001 & & 0.0001 \\
\hline 0 & 38 & 96.7 & & 78.6 & \\
\hline 1 & 18 & 88.2 & & 88.2 & \\
\hline 2 & 20 & 49.9 & & 35.1 & \\
\hline 3 & 29 & 30.2 & & 30.2 & \\
\hline Lymphatic invasion (ly) & & & 0.0168 & & 0.0342 \\
\hline 0 & 44 & 79.9 & & 74.7 & \\
\hline 1 & 24 & 68.7 & & 59.9 & \\
\hline 2 & 16 & 67.5 & & 50.0 & \\
\hline 3 & 21 & 39.7 & & 41.3 & \\
\hline Venous invasion (v) & & & 0.0058 & & 0.0027 \\
\hline 0 & 61 & 79.9 & & 77.9 & \\
\hline 1 & 30 & 51.4 & & 39.3 & \\
\hline 2 & 11 & 33.8 & & 33.8 & \\
\hline 3 & 3 & 66.7 & & 50.0 & \\
\hline Histology & & & 0.4996 & & 0.6319 \\
\hline tub & 40 & 75.2 & & 67.3 & \\
\hline por & 55 & 61.8 & & 57.0 & \\
\hline Other & 10 & 66.7 & & 60.0 & \\
\hline Tumor infiltrative type (IFN) & & & 0.0007 & & 0.0026 \\
\hline $\mathrm{a}$ & 28 & 79.6 & & 63.0 & \\
\hline $\mathrm{b}$ & 35 & 74.9 & & 62.6 & \\
\hline c & 30 & 34.7 & & 36.1 & \\
\hline Lauren classification & & & 0.1586 & & 0.042 \\
\hline $\mathrm{i}$ & 40 & 61.9 & & 52.8 & \\
\hline $\mathrm{m}$ & 32 & 76.0 & & 56.7 & \\
\hline d & 21 & 46.4 & & 61.4 & \\
\hline
\end{tabular}

OS overall survival, RFS recurrence-free survival

positive group exhibited lower RFS rate than TMPRSS4negative group (Fig. S1a, b). Also, there was no significant difference in OS and RFS rates of patients with stages II GC. (Fig. S1c, d). In regard to stage IV, the number of patients at stage IV was 10 , among which there was only 2 TMPRSS4-negative cases, which was too small to analyze the OS. 
Table 3 Multivariate analyses of OS and RFS in patients with gastric cancer

\begin{tabular}{|c|c|c|c|c|c|c|}
\hline & & OS & & & RFS & \\
\hline & HR & $95 \% \mathrm{CI}$ & $p$ value & HR & $95 \% \mathrm{CI}$ & $p$ value \\
\hline Venous invasion & 2.79 & $1.150-3.740$ & 0.0016 & & & \\
\hline pStage & 1.94 & $0.016-2.922$ & 0.012 & 2.03 & $1.295-6.892$ & 0.0094 \\
\hline TMPRSS4 & & & & 2.89 & -1.3314 to -0.2897 & 0.0013 \\
\hline
\end{tabular}

$O S$ overall survival, $R F S$ recurrence-free survival, $H R$ hazard ratio, $C I$ confidence interval

\section{Silencing of TMPRSS4 Reduces the Migration of GC Cells and Enhances the Sensitivity to 5-FU}

Western blot analysis revealed that TMPRSS4 protein was differentially expressed in both NUGC-3 and MKN-45 cells (Fig. 4a, S2a). The delivery of TMPRSS4-targeting siRNA led to a significant decrease in mRNA and protein levels of TMPRSS4 in both MKN-45 and NUGC-3 cells (Fig. 4a, S2a). Cell proliferation assay revealed that TMPRSS4-silencing did not reduce the proliferation of the two GC cell lines. However, the wound-healing assay revealed that TMPRSS4-silencing significantly suppressed the wound healing when compared to the control siRNA-transfected cells $(p=0.03)$ (Fig. 4b, S2b).

Thereafter, we tested the effect of 5-FU on GC cells. TMPRSS4-silenced NUGC-3 and MKN-45 cells were found to be highly and significantly sensitive to 5-FU compared to the corresponding non-specific control siRNA-transfected cells (Fig. 4c, S2c).

a

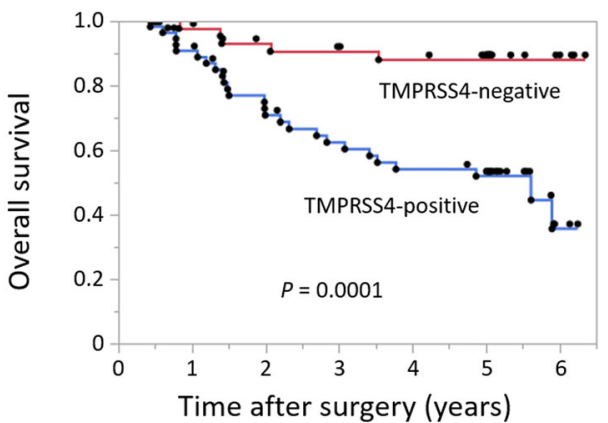

b

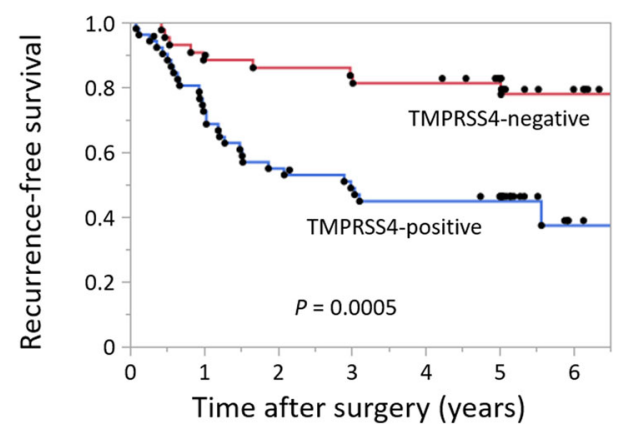

Fig. 2 Kaplan-Meier curves for all GC patients classified based on the expression of TMPRSS4. a Overall survival (OS) curves of all GC patients. b Recurrence-free survival (RFS) curves of all GC patients. The OS and RFS outcomes were compared using the log-rank test.

\section{Discussion}

In the present study, we evaluated whether the expression of TMPRSS4 is associated with the clinico-pathological factors and the outcomes after gastrectomy for the patients with GC. Additionally, we examined the effect of TMPRSS4 expression in vitro on the proliferation, migration, and the sensitivity to chemotherapy of GC cells. Our results showed that the expression of TMPRSS4 is significantly correlated with deeper tumor and venous invasion. Moreover, GC patients with positive expression of TMPRSS4 exhibited poor RFS. Especially, in the subgroup of patients with stage III GC, the positive expression of TMPRSS4 was significantly correlated with poor OS and RFS after gastrectomy, as compared to the negative expression of TMPRSS4. Low expression of TMPRSS4 suppressed the migration of GC cells, but not the proliferation. However, low expression of TMPRSS4 in GC cells enhanced their sensitivity to 5-FU. These results suggest

a

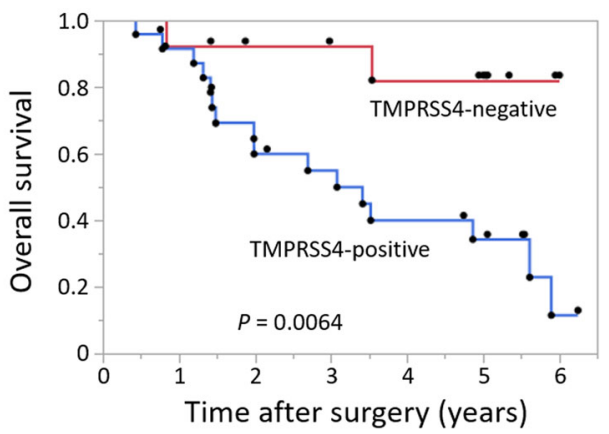

b

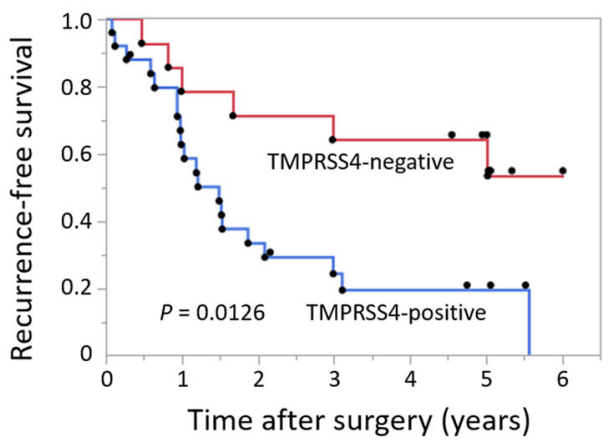

Fig. 3 Kaplan-Meier curves for all GC patients classified based on the expression of TMPRSS4 in stage III. a OS curves of GC patients in stage III. b RFS curves of GC patients in stage III. The OS and RFS were compared using the log-rank test. 

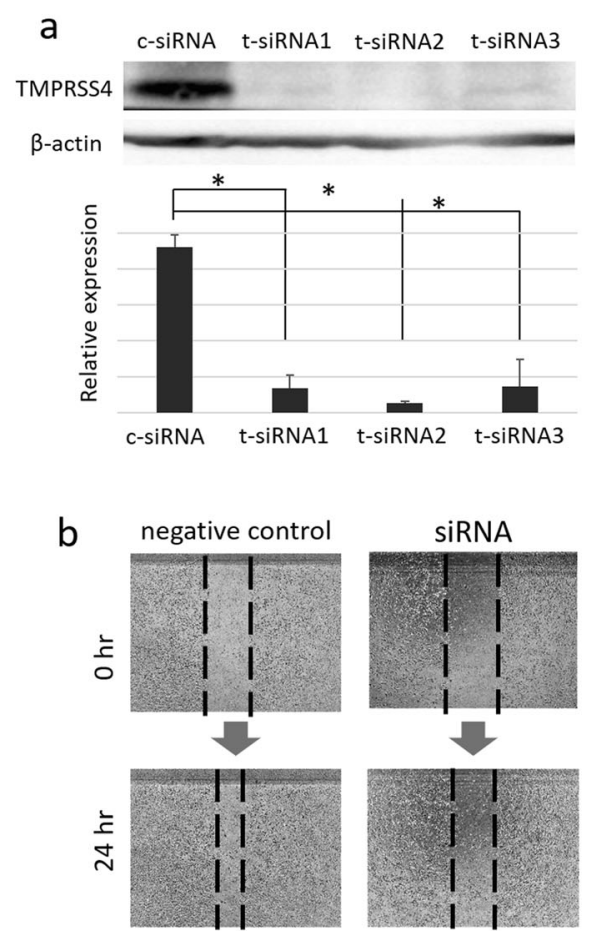

Fig. 4 TMPRSS4-silencing reduces the migration and chemosensitivity to 5-FU in GC cells. a mRNA levels of TMPRSS4 in NUGC-3 cells transfected with control siRNA (c-siRNA) or TMPRSS4-targeting siRNA (t-siRNA). Control siRNA transfected cells were used as control. Western blot analysis of TMPRSS4 protein levels in NUGC-3 cells transfected with control siRNA (c-siRNA) or TMPRSS4-targeting siRNA ( $\mathrm{t}$-siRNA). Control siRNA transfected cells were used as control.

that TMPRSS4 is a potential prognostic factor for the recurrence of $\mathrm{GC}$ and, thus, can be considered as a promising therapeutic target.

Several studies have been conducted to identify the useful biomarkers for predicting the outcomes of GC patients at stages II and III. ${ }^{19}-25 \mathrm{Kim} \mathrm{MH}$. et al. focused on thymidylate synthetase (TS), which influences the response to fluoropyrimidine-based chemotherapy, including TS-1 and capecitabine. ${ }^{24}$ In classical trial, they showed that high TMPRSS4 expression is a predictive factor for the worse outcomes during capecitabine plus oxaliplatin adjuvant chemotherapy. ${ }^{26},{ }^{27}$ However, the number of patients with high TMPRSS4 expression was small (15.8\%), and there was no significant difference in the outcomes between stages II and III patients; however, stage III patients did show shorter OS and DFS than those at stage II. ${ }^{24}$ There were several reports regarding programmed cell death ligand-1 (PD-L1) in GC. ${ }^{24}, 25$ However, there were no association between the expression of PD-L1 and the outcomes in adjuvant chemotherapy subgroup of stages II and III patients. Kikuchi et al. using proteome analyses showed that the overexpression of ephrin A2 receptors in cancer stromal cells act as a prognostic factor for the relapse of GC in patients with S-1 adjuvant chemotherapy.
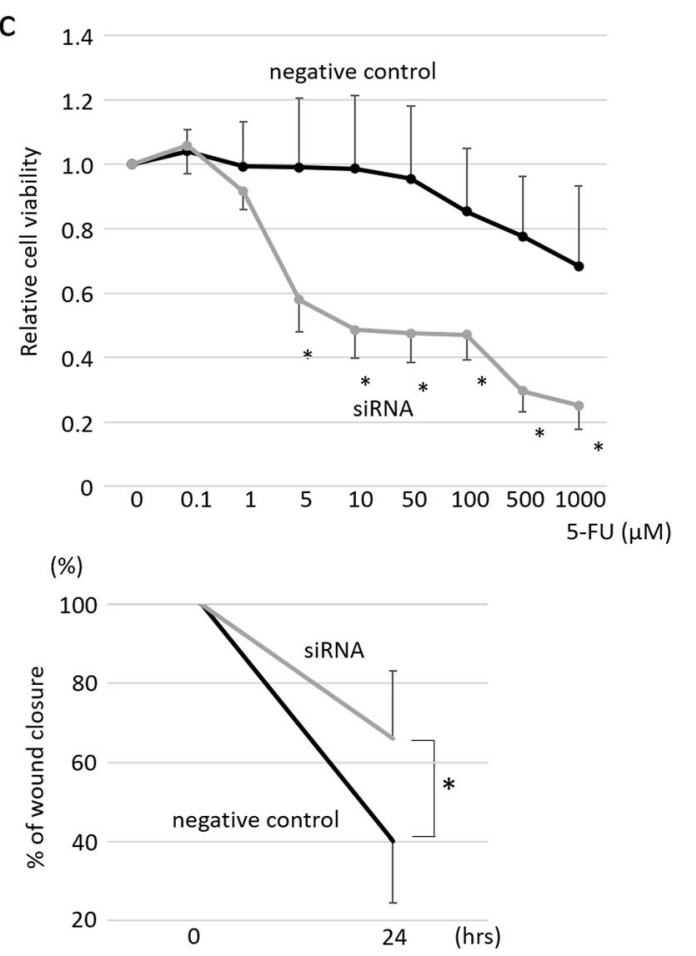

Bar graphs indicate densitometric analysis of western blots from three independent experiments. (1, negative control; 2, TMPRSS4-targeting siRNA \#1; 3, TMPRSS4-targeting siRNA \#2; and 4, TMPRSS4targeting siRNA \#3). b Representative images of the migrated cells taken at $24 \mathrm{~h}$ after scratching. Bar graphs indicate the mean percentage $( \pm$ SEM) of wound closure. $\mathbf{c}$ Proliferation assay using CCK-8 kit were performed to examine the inhibitory effect of 5-FU. ${ }^{*} p<0.05$

This biomarker could be promising; however, there was no significant difference noted in OS, and the authors stated that the number of patients was too small to indicate a statistical difference. Our multivariate analysis showed that TMPRSS4 was a significant prognostic factor for the recurrence in all GC patients who underwent curative operation. This result indicates that TMPRSS4 can be applied as a useful biomarker for the recurrence in patients undergoing curative operation. We think that TMPRSS4 exhibits the advantage of high expression rate, because the number of patients with high TMPRSS4 expression (56.3\%) was high in our case. However, our study is single institutional, and the number of patients is also small. Therefore, a largescale study is needed to validate the usefulness of TMPRSS4 as a biomarker for successful prognosis of GC. Our results are in accordance with the previous report published by Sheng. et al. ${ }^{16}$ They showed that the 5-year survival rates of TMPRSS4-positive and negative patients with stages II and III GC were approximately $40 \%$ and $80 \%$, respectively, further proposing that TMPRSS4 expression can be applied as an indicator of poor prognosis in GC patients. However, this study did not include the adjuvant chemotherapy. In our study, all GC patients at stages II and III received adjuvant S-1 monotherapy. 
Our results showed that the 5-year OS and RFS rates of TMPRSS4-negative patients with stage III GC were significantly higher than those of TMPRSS4-positive patients with stage III GC. The downregulation of TMPRSS4 induced an increased susceptibility to 5-FU. These results suggest the possibility that TMPRSS4-negative expression increases chemo-sensitivity, resulting in the suppression of recurrence in TMPRSS4-negative GC patients at stage III. ACTS-GC trial demonstrated that the 5-year OS rate in patients with stage IIIB GC undergoing S-1 adjuvant chemotherapy was 50.2\%, suggesting that it can be improved further. ${ }^{3}$ Therefore, the addition of docetaxel to S-1 adjuvant chemotherapy, in JACCROGC-07 trial, improved the 3-year RFS rate in patients with stage III GC, compared with S-1 alone, although the data related to OS was not presented. ${ }^{4}$ In Japan, S-1 plus docetaxel replaced S-1 monotherapy in patients with stage III GC since it was published. However, S-1 plus docetaxel therapy likely induces grade 3 or greater adverse events, such as neutropenia and alopecia, compared with S-1 alone adjuvant therapy. Therefore, the expression of TMPRSS4 may be a useful predictor for modulating the S-1 plus docetaxel therapy in patients with stage III GC, because the prognosis of TMPRSS4- negative patients at stage III was notable under only S- 1 monotherapy, as the 5-year survival rate was $85 \%$.

In the present study, we showed that low expression of TMPRSS4 suppressed the migration of GC cells and enhanced the sensitivity to 5-FU. These results were in accordance with the previous reports. ${ }^{9} 28$ Exposito et al. showed using lung cancer cell lines that the downregulation of TMPRSS4 could sensitize cells to the cytotoxic effect of several chemotherapy drugs, including 5-FU. The knockdown of TMPRSS4 in lung cancer cells induced the downregulation of thymidylate synthetase (TS). TS catalyzes the conversion of dUMP to dTMP, which is required for DNA synthesis and repair. Basically, high TS expression has been shown to result in relatively low sensitivity to fluoropyrimidine-based chemotherapy, including S-1. ${ }^{29}, 30$ Downregulation of TMPRSS4 may sensitize cancer cells to the cytotoxic effects of S-1 via decreased expression of TS. Conversely, Sasako et al. showed a significant association between high TS expression and an enhanced outcome in ACTS-GC. ${ }^{20}$ Therefore, it is required to conduct further studied to clarify this discrepancy.

In the subgroup of patients with stage II GC, there was no significant difference in the OS between the positive and negative expression of TMPRSS4, although the OS of GC patients with negative expression of TMPRSS4 was higher. The number of patients in the subgroup of stage II GC was small. Therefore, it is required to conduct a study with large sample size to validate the impact of TMPRSS4 on stage II GC.

TMPRSS4 is considered as a novel potential therapeutic target in GC. ${ }^{31}$ Our study showed that low expression of TMPRSS4 suppressed the migration of GC cells and enhanced the sensitivity to 5 -FU. These results suggest that TMPRSS4 may be considered as a good target candidate. The pharmacological compounds to inhibit TMPRSS4 in combination with chemotherapy, such as S-1, may promote the sensitivity to chemotherapy through inhibiting the function of TMPRSS4. Recently, 2-hydroxydiarylamide derivatives, IMD-0354 and KRT1853, were developed as TMPRSS4 serine protease inhibitors. ${ }^{32}$ These new compounds have been shown to inhibit cancer cell invasion, migration, and proliferation; however, the sensitivity to chemotherapy was not determined.

In conclusion, TMPRSS4 can be considered as a potential prognostic biomarker for GC, especially in stage III, and a promising therapeutic target.

Supplementary Information The online version contains supplementary material available at https://doi.org/10.1007/s11605-021-05101-2.

Acknowledgments We thank Mrs. Ishida for her contribution in maintaining the cell cultures and Mrs. Shitakubo for her contribution in conducting the statistical analyses.

\section{Declarations}

Statement of Informed Consent Written informed consent was obtained from all of the participants involved in this study. All procedures were accepted by Kure Medical Center - Chugoku Cancer Center.

Conflict of interest The authors declare no competing interests.

Open Access This article is licensed under a Creative Commons Attribution 4.0 International License, which permits use, sharing, adaptation, distribution and reproduction in any medium or format, as long as you give appropriate credit to the original author(s) and the source, provide a link to the Creative Commons licence, and indicate if changes were made. The images or other third party material in this article are included in the article's Creative Commons licence, unless indicated otherwise in a credit line to the material. If material is not included in the article's Creative Commons licence and your intended use is not permitted by statutory regulation or exceeds the permitted use, you will need to obtain permission directly from the copyright holder. To view a copy of this licence, visit http://creativecommons.org/licenses/by/4.0/.

\section{References}

1. Paoletti X, Oba K, Burzykowski T, et al. and the GASTRIC (Global Advanced/Adjuvant Stomach Tumor Research International Collaboration) Group. Benefit of adjuvant chemotherapy for resectable gastric cancer: a meta-analysis. JAMA 2010;303(17): 17291737.

2. Sakuramoto S, Sasako M, Yamaguchi T, et al. Adjuvant chemotherapy for gastric cancer with S-1, an oral fluoropyrimidine. N Eng J Med 2007; 357(18):1810-20. 
3. Sasako M, Sakuramoto S, Katai H, et al. Five-year outcomes of a randomized phase III trial comparing adjuvant chemotherapy with S-1 versus surgery alone in stage II or III gastric cancer. J Clin Oncol 2011; 29(33): 4387-4393.

4. Yoshida K, Kodera Y, Kochi M, et al. Addition of docetaxel to oral fluoropyrimidine improves efficacy in patients with stage III gastric cancer: interim analysis of JACCRO GC-07, a randomized controlled trial. J Clin Oncol 2019; 37(15): 1296-1304.

5. Tanabe L. List K. The role of type II transmembrane serine protease-mediated signaling in cancer. FEBS 2017; 284(10): 1421-1436.

6. Martin CE, List K. Cell-surface anchored serine proteases in cancer progression and metastasis. Cancer Metastasis Rev 2019; 38(3): 357-387.

7. Larzabal L, Nguewa PA, Pio R, et al. Over expression of TMPRSS4 in non-small cell lung cancer is associated with poor prognosis in patients with squamous histology. Br J Cancer 2011; 105(10): 1608-1614.

8. Luo ZY, Wang YY, Zhao ZS, Li B Chen JF. The expression of TMPRSS4 and Erk1 correlates with metastasis and poor prognosis in Chinese patients with gastric cancer. Plos One 2013; 8(7): 70311

9. Expsito T, Villalba M, Redrado M, et al. Targeting of TMPRSS4 sensitizes lung cancer cells to chemotherapy by impairing the proliferation machinery. Cancer lett 2019; 453: 21-33.

10. Li XM, Liu WL, Chen X, et al. Overexpression of TMPRSS4 promotes tumor proliferation and aggressiveness in breast cancer. Int J Mol Med 2017; 39(4): 927-935.

11. Cheng Y, Wang K, Geng L, et al. Identification of candidate diagnostic and prognostic biomarkers for pancreatic carcinoma. EBiomedicine 2019; 40: 382-393.

12. Dong ZR, Sun D, Yang YF, et al. TMPRSS4 drives angiogenesis in hepatocellular carcinoma by promoting HB-EGF expression and proteolytic cleavage. Hepatology 2020; 72(3): 923-939.

13. Jung H, Lee KP, Park SJ, et al. TMPRSS4 promotes invasion, migration, and metastasis of human tumor cells by facilitating an epithelial-mesenchymal transition. Oncogene 2008; 27(18): 26352647.

14. Kim S, Kang HY, Nam EH, et al. TMPRSS4 induces invasion and epithelial-mesenchymal transition through upregulation of integrin $\alpha$ and its signaling pathways. Carcinogenesis 2010; 31(4): 597-606.

15. Min HJ, Lee MK, Lee JW, Kim S. TMPRSS4 induces cancer cell invasion through pro-uPA processing. Biochem Biophys Res Commun 2014; 446(1): 1-7.

16. Sheng H, Shen W, Zeng J, Xi L, Deng L. Prognostic significance of TMPRSS4 in gastric cancer. Neoplasma 2014; 61(2): 213- 217.

17. Japanese Gastric Cancer Society. Guideline for Diagnosis and Treatment of Carcinoma of the Stomach.

18. Japanese classification of gastric carcinoma. $3^{\text {rd }}$ English edition. Gastric Cancer.2011;14(2): 101-112.

19. Terashima M, Kitada K, Ochiai A, et al. Impact of expression of human epidermal growth factor receptors EGFR and ERBB2 on survival in stage II/II gastric cancer. Clin Cancer Res 2012; 18(21): 5992-6000.
20. Sasako M, Terashima M, Ichikawa W, et al. Impact of the expression of thymidylate synthase and dihydropyrimidine dehydrogenase genes on survival in stage II/III gastric cancer. Gastric Cancer 2015; 18(3): 538-548.

21. Kikuchi S, Kaibe N, Morimoto K, et al. Over expression of ephrin A2 receptors in cancer stromal cells is a prognostic factor for the relapse of gastric cancer. Gastric Cancer 2015; 18(3): 485-494.

22. Terashima M, Ichikawa $\mathrm{W}$, Ochiai $\mathrm{A}$, et al. TOP2A, GGH, and PECAM1 are associated with hematogeneous, lymph node, and peritoneal recurrence in stage II/III gastric cancer patients enrolled in the ACRS-GC study. Oncotarget 2017; 8(34): 57574-57582.

23. Ichikawa $\mathrm{W}$, Terashima $\mathrm{M}$, Ochiai $\mathrm{A}$, et al. Impact of insulin-like growth factor- 1 and amphiregulin expression on survival in patients with stage II/III gastric cancer enrolled in the adjuvant chemotherapy trial of S-1 for gastric cancer. Gastric Cancer 2017; 20(2): 263273.

24. Kim MH, Zhang X, Jung M, Jung I, Park HS, Beom SH, Immunohistochemistry biomarkers predict survival in stage II/III gastric cancer patients: from a prospective clinical trial. Cancer Res Treat 2019; 51(2): 819-831.

25. Choi YY, Kim H, Shin SJ, et al. Microsatellite instability and programmed cell death-ligand 1 expression in stage II/III gastric cancer: post hoc analysis of the CLASSIC randomized controlled study. Ann Surg 2019; 270(2): 309-316.

26. Bang YJ, Kim YW, Yang HK, et al. Adjuvant capecitabine and oxaliplatin for gastric cancer after D2 gastrectomy (CLASSIC): a phase 3 open-label, randomized controlled trial. Lancet 2012; 379(9813): 305-321.

27. Nah SH, Park SR, Yang HK, et al. Adjuvant capecitabine plus oxaliplatin for gastric cancer after D2 gastrectomy (CLASSIC): 5year follow-up of an open-label, randomized phase 3 trial. Lancet Oncol 2014; 15(12): 1389-1396.

28. Jin J, Shen X, Chen L, Bao LW, Zhu LM. TMPRSS4 promotes invasiveness of human gastric cancer cells through activation of NF- $\alpha$ B/MMP-9 signaling. Biomedicine and Pharmacotherapy 2016; 77: 30-36.

29. Takezawa K, Okamoto I, Okamoto W, et al. Thymidylate synthase as a determinant of pemetrexed sensitivity in non-small cell lung cancer. Br J cancer. 2011; 104(10): 1594-1601.

30. Wada Y, Yoshida K, Suzuki T, et al. Synergetic effects of docetaxel and S-1 by modulating the expression of metabolic of 5-fluorouracil in human gastric cancer cell lines. Int J Cancer 2006; 119(4): 783791.

31. Aberasturi AL, Calve A. TMPRSS4: an emerging potential therapeutic target in cancer. Br J Cancer 2015; 112(1): 4-8.

32. Kim S, Ko D, Lee Y, et al. Anti-cancer activity of the novel 2hydroxydiarylamide derivatives IMD-0354 and KRT1853 through suppression of cancer cell invasion, proliferation, and survival mediated by TMPRSS4. Sci Rep 2019; 9(1): 100003.

Publisher's Note Springer Nature remains neutral with regard to jurisdictional claims in published maps and institutional affiliations. 